\title{
A Robust Embedded Non-Linear Acoustic Noise Cancellation (ANC) Using Artificial Neural Network (ANN) for Improving the Quality of Voice Communications
}

\author{
Azeddine Wahbi ${ }^{\mathrm{a}}$, Ahmed Roukhe ${ }^{\mathrm{b}}$, Bahloul Bensassi ${ }^{\mathrm{a}}$, Laamari Hlou ${ }^{\mathrm{c}}$ \\ ${ }^{a}$ Laboratory of Industrial Engineering, Information Processing and Logistic,Faculty of sciences Ain Chock, Hassan II University, \\ Casablanca, Morocco \\ ${ }^{b}$ Laboratory of Atomic, Mechanical, Photonics and Energy Faculty of Science, University Moulay Ismail, Meknes, Morocco \\ ${ }^{c}$ Laboratory of Electronic Systems, Information Processing and Energetics, Faculty of Sciences, University Ibn Tofail Kenitra, Morocco \\ Corresponding author: wahbi_azeddine@yahoo.fr
}

\begin{abstract}
Embedded Acoustic Noise Cancellation (ANC) has enjoyed remarkable success in the telecommunication field, and it becomes an essential component in various communications applications, such as digital transmission. So, it is an efficient method used to enhance the quality of communications against noise phenomena which is a problem in communication systems. This paper contributes towards a new non-linear embedded ANC based Artificial Neural Network (ANN) in digital signal processing and backpropagation (BP) of the gradient algorithm. This system is usually required for non-linear adaptive processing digital signals. The neuronal ANC estimates the noise path and subtracting noise from a received signal by minimizing a cost function. It is the mean square error. Thus, also the filter weights are adaptively updated. In this work, we designed and simulated our intelligent embedded ANC model with the help of MATLAB\Simulink software. The proposed system was designed by using embedded functions in Simulink. In addition, all simulation results are performed and verified using Signal Noise to Ratio (SNR) and Mean Square Error (MSE), number of iteration, neuronal architecture, criteria and it has been compared in various scenarios. Finally, a study and analysis on convergence of neuronal ANC based backpropagation of the gradient algorithm demonstrate that our proposed system can effectively improve the quality of voice communications against the undesired noise. It also provides faster convergence during the back propagation of the gradient. Furthermore, the best values of SNR and MSE show the effectiveness of the proposed model.
\end{abstract}

Keywords - Embedded systems; noise cancellation problem; artificial neural network; digital signal processing; back propagation.

\section{INTRODUCTION}

ANC is a system that has been widely applied for various purposes, especially in communication systems. It has many advantages for eliminating the unwanted acoustic noise that affects the quality of voice communications [1]-[3]. At the same time, neural networks, digital signal processing and embedded systems have become increasingly important in many applications such as compression, classification, identification, and recognition, and Noise cancellation [4], [5]. It is also very widely that acoustic noise cancellation in voice communications has used this efficient technology. In this case, the recent technology of ANC should use the new approaches based on artificial neural networks.
There have been numerous efforts to develop more adaptive approaches to enhance the quality of communication [6]-[12]. Wahbi et al. implemented an ANC by using adaptive filters in time and frequency domain to remove acoustic noise from voice communications [13]-[15]. In each module, we implemented and tested the behavior of ANC according to the international recommendations. Also, many classical systems are developed to have enhanced the quality of communications against acoustic noise using adaptive filters [16]-[18].

Several types of neural networks have been developed. Maximilian Strake et al. [19] have introduced a fully convolutional recurrent networks for speech enhancement. It is able to preserve the local structure of spectral harmonics present in the features at its input. In addition, ANC based Recurrent Neural Networks are employed for Speech 
enhancement [20]-[22]. In this paper, we present a new algorithm for noise cancellation using non-linear ANN approach. We focus our study on the type MLP. In the process of building this type of acoustic noise canceller, learning aims to adapt the weights of connections between neurons so that the network will output the enhanced signal with the same form as the desired input. That is to minimize the error of the network on all forms of learning base. However, the Embedded ANC is developed using a Multi-layer Perception (MLP) network, a feedforward neural network trained with the backpropagation algorithm. The main scope of this approach is the fast convergence during the learning phase.

\section{ANN FOR NOISE CANCELLING}

\section{A. ANN Filters for Noise Cancellation}

Fig. 1 describes its structure where the desired response is composed of an original signal plus the noised, which is uncorrelated with the signal. The filter input is a sequence of a noised signal which is correlated with the noised signal in the desired signal. By using the ANN algorithm inside the adaptive filter, the error term $\mathrm{e}_{\mathrm{n}}$ produced by this system is then the original signal with the noise signal canceled. The output signal network to each input signal is directly compared with the desired signal and a feedback is given to the network to correct possible signal errors.

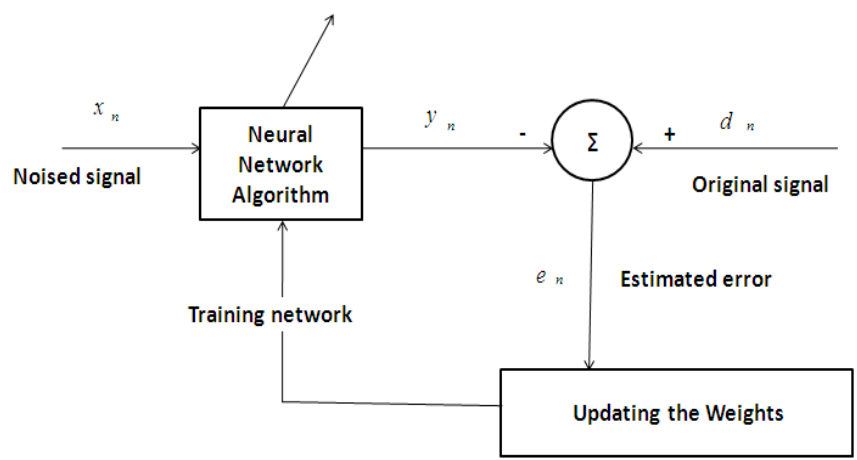

Fig. 1 BP ANN filter structure

\section{B. MLP Network Architecture}

1) Description of Artificial Neural Networks: The interconnection of neurons forms a network. Neurons are arranged in layers: an input layer, an output layer, and one or more hidden layers between the input and output layers. However, the model used in our developed ANC is a Multilayer Perception based (MLP) network (Fig. 2). They are generally characterized by:

- One input layer representing inputs which are transmitted the data to be processed from a source external to the network

- .One or more hidden layers performing the specific treatment of the network.

- An output that delivers results.

The inputs to a neuron include its bias and the sum of its weighted inputs (Fig. 3). A neuron's output depends on the neuron's inputs and on its transfer function [23]. There are many useful transfer functions or activation functions.

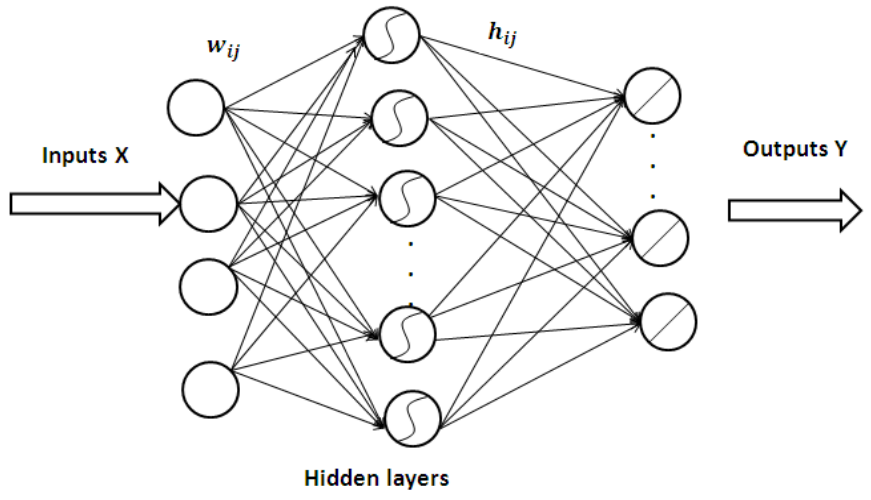

Fig. 2 MLP architecture using supervised learning.

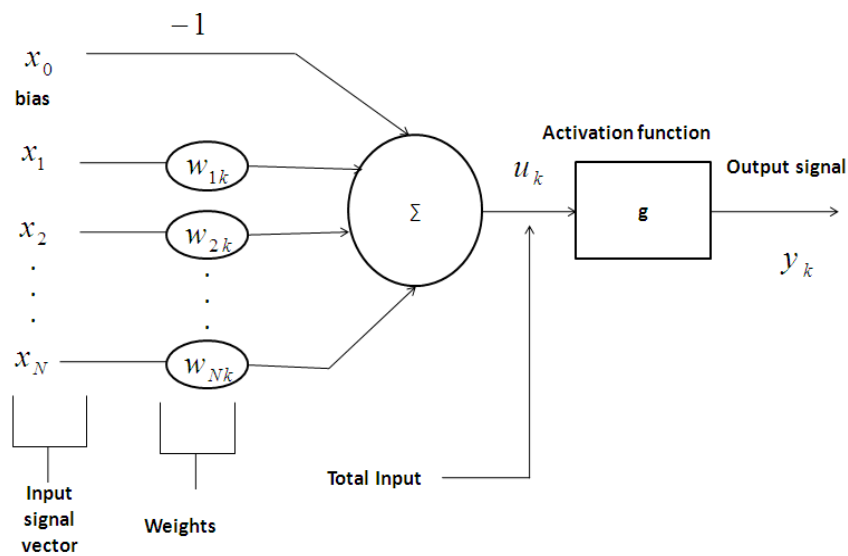

Fig. 3 Schematic representation of a neuron

2) ANN algorithm for modeling Embedded ANC System formulation: Table I depicts a summary of developed Embedded ANC System-based ANN variables.

TABLE I

VARIABLES OF ANN ANC MODEL

\begin{tabular}{cl}
\hline Variable & \multicolumn{1}{c}{ Description } \\
\hline $\mathrm{x}_{\mathrm{n}}$ & $\begin{array}{l}\text { Noised signal in time domain, at } \\
\text { each discrete instant } \mathrm{n}\end{array}$ \\
$\mathrm{yn}$ & $\begin{array}{l}\text { Filtered signal in time domain, at } \\
\text { each discrete instant } \mathrm{n} \\
\text { Original signal provided to the } \\
\text { network in time domain, at each } \\
\text { discrete instant } \mathrm{n}\end{array}$ \\
$\mathrm{d}_{\mathrm{n}}$ & $\begin{array}{l}\text { Estimated error in time domain, at } \\
\text { each discrete instant } \mathrm{n}\end{array}$ \\
\hline
\end{tabular}

3) Back Propagation of Gradient: The adjustment of the weight's connection and biases of different layers can be obtained by minimizing a cost function using the back propagation gradient [24]. The cost function, Mean Square Error (MSE), is a function of the difference between a desired output and the actual output of the ANN filter. This difference is known as the estimation error of the ANN algorithm.

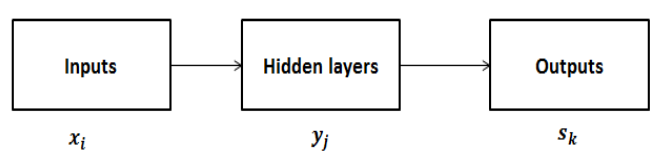

Fig. 4 Simplified diagram of MLP

Each iteration " $k$ " of the ANN ANC requires below distinct steps in this order: 
- The output of hidden layer, maybe calculated by using the following equation:

$$
y_{j}=g_{1}\left(\sum_{i} x_{i} w_{i j}\right)=g_{1}\left(b_{j}\right)
$$

Where, $b_{j}$ : is the total input of hidden layer $y_{j}$.

- The final output of network, which represents the noise estimated, maybe calculated by using the following equation:

$$
s_{k}=g_{2}\left(\sum_{j} y_{j} h_{j k}\right)=g_{2}\left(a_{k}\right)
$$

Where, $a_{k}$ : is the total input of output hidden $s_{k}$.

- The error signal have often calculated as :

$$
e_{k}=d_{k}-s_{k}
$$

Firstly, the cost function (C), which will minimize, is the Mean Square Error (MSE) defined by:

$$
C\left(h_{k}\right)=\frac{1}{2} \sum_{k}\left(d_{k}-s_{k}\left(h_{k}\right)\right)^{2}
$$

The partial derivative of cost function $C$, over $h_{j k}$, represents the variation of error according to weights variation.

On all training, we have:

$$
\frac{\partial C\left(h_{j k}\right)}{\partial h_{j k}}=-\sum_{k}\left(d_{k}-s_{k}\right) \frac{\partial s_{k}}{\partial h_{j k}}
$$

The $h_{j k+1}$ and $w_{j k+1}$ vectors for updating weights can be written by the following expressions:

$$
\begin{gathered}
h_{j k+1}=h_{j k}-\eta_{1}\left(\sum_{k} \delta_{2}\right) y_{j} \\
w_{j k+1}=w_{j k}-\eta_{2} \sum_{j}\left(\sum_{k} \delta_{1} y_{l}\left(1-y_{k}\right)\right) x_{j}
\end{gathered}
$$

We denote $\delta_{2}$ and $\delta_{1}$ propagation errors for the output layer and the output of the hidden layer.

Each iteration of the BP ANN algorithm requires 8 distinct steps in the following order:

Step 1: Initialize the weights.

Step 2: Define the input vector and corresponding desired output.

Step 3: Calculate the network output. (Eq.1)

Step 4: Calculate the error in the output. (Eq.3)

Step 5: Calculate the gradient of the error with respect to weights. (Eq.5)

Step 6: Update the filter tap weights. (Eq.6 and Eq.7)

Step 7: Re-inject the output error in the network and calculate the error in hidden layers. -if the condition on the error or number of iteration is matched, go to step 8, else apply the new input vector and go to step 3.

Step 8: end.

\section{Modeling AND Simulation Results}

\section{A. Model of Proposed ANN Embedded ANC Algorithm}

An ANN Embedded Noise Canceller developed to reduce noise in voice communications is presented in Fig. 5.

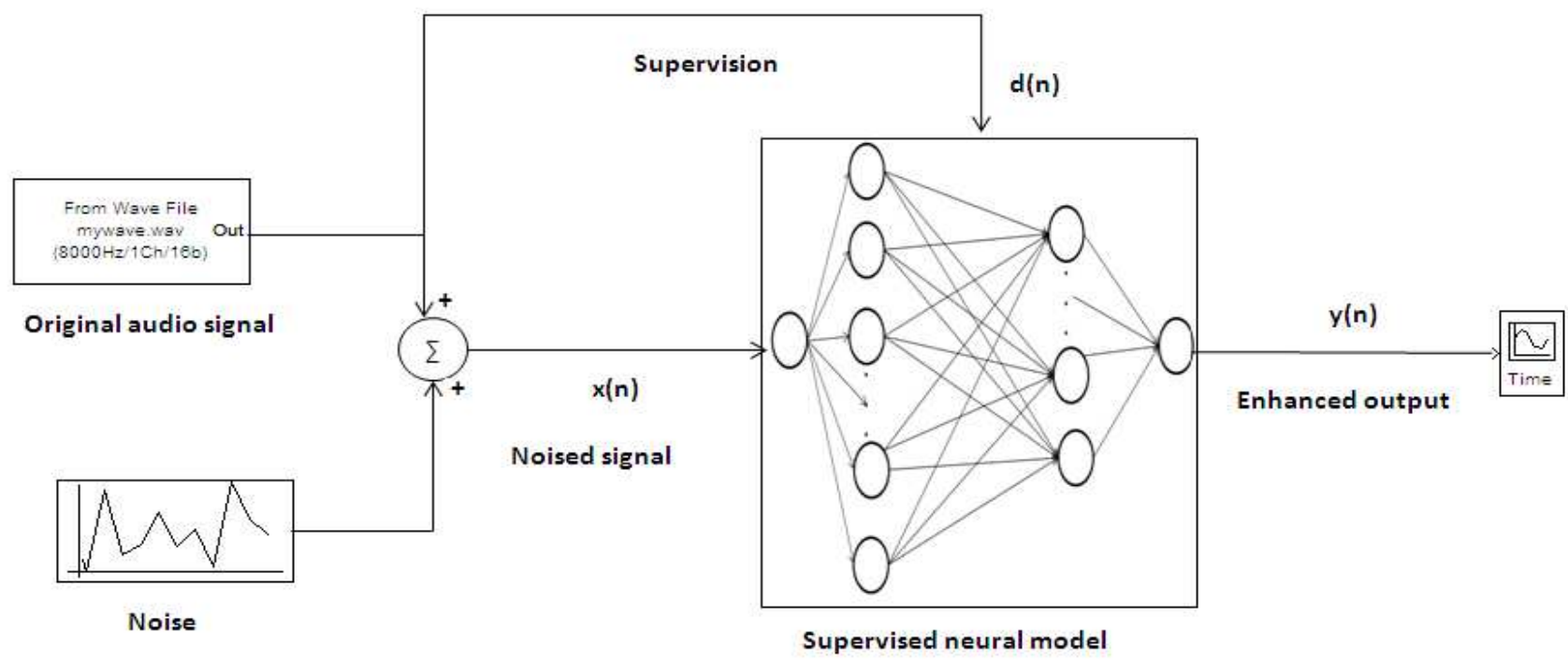

Fig. 5 ANN Embedded ANC model

In this work, we modeled and developed the system shown in Fig. 5 under Matlab by creating and programming our new embedded functions with the following specifications: The number of samples for original signal length is 256 samples per frame $8000 \mathrm{~Hz}$ sampling rate. ANN ANC implementation is set up with inputs and outputs signals length $=256$. It includes noise, original signal, desired input, ANN output, enhanced output. $\mathrm{SNR}=29.4823$. The noise type is Gaussian with zero mean and one variance.

\section{B. Flow chart of ANN ANC Algorithm}

Modeling ANN Embedded ANC system may perform according to the following steps as shown in Fig. 6. 


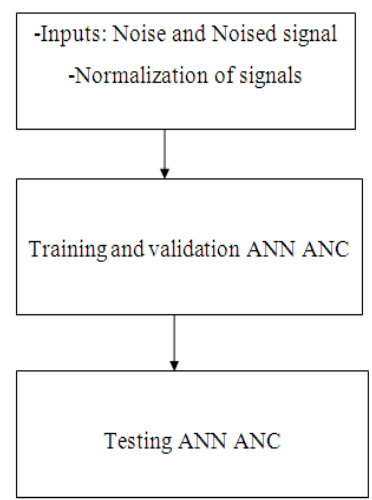

Fig. 6 ANN Embedded ANC System flowchart

In this work, tangent sigmoid and linear activation functions will be used respectively in the hidden layers and the output layer. Those functions are defined respectively by the equations (8) and (9):

$$
\begin{gathered}
g(u)=\frac{1}{1+e^{-a u}} \\
y_{k}=g(u)=\left\{\begin{array}{l}
1 \text { siu }_{k} \geq 0 \\
0 \text { siu }_{k} \prec 0
\end{array}\right.
\end{gathered}
$$

For defining the number of hidden layers and the neurons in each layer, the designer must have a great number of experiences. For example, we were varying the network size, and we do a full training for each size and finally choose the structure that provides the best results.

\section{Simulation Results}

According to the neural model, our simulation modeling and design results are obtained after a number of tests of iterations by acting respectively on the number of hidden layers, the number of neurons per hidden layers and network settings. All measurements are taken using a LevenbergMarquardt type of learning. Fig. 7 shows the best performance for the validation of the neural system of acoustic noise cancellation based on the number of iterations adequate. Learning stops when the mean squared error (MSE) based on the number of samples starts to decrease to less than a certain threshold, knowing that the MSE criterion represents the standard deviation between the filtered signal and the desired signal.

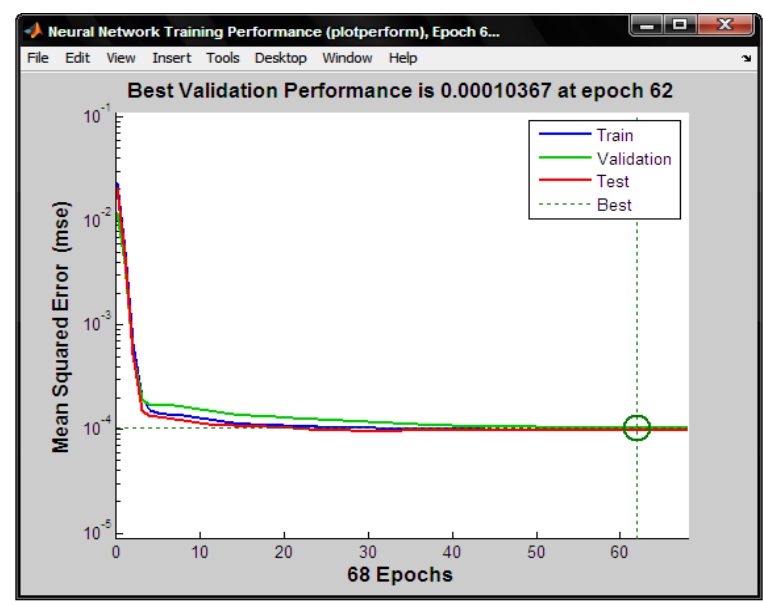

Fig. 7 Performance of neural ANC based Levenberg-Marquardt's algorithm

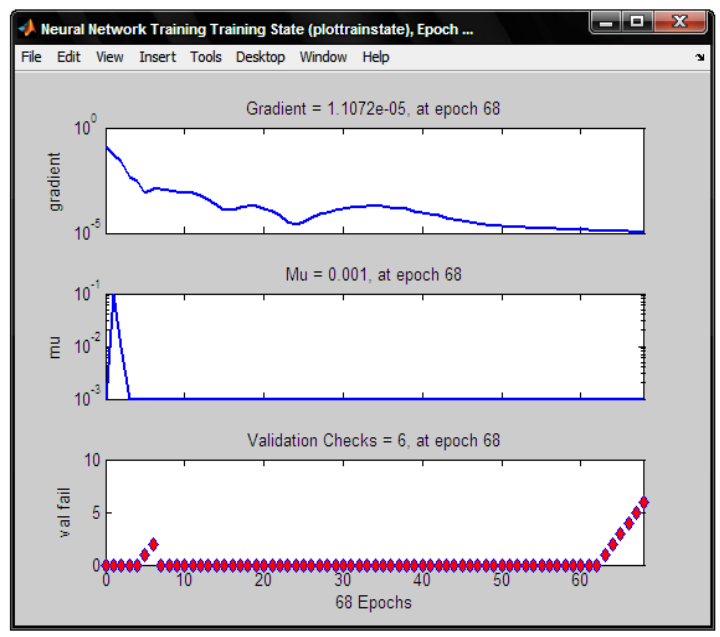

Fig. 8 ANC, gradient Evolution, $\mathrm{Mu}$, and validation number of iteration

Fig. 8 illustrates the better performance of the backpropagation algorithm during the learning phase. This value is equal to 0.00010367 at 62 iterations.

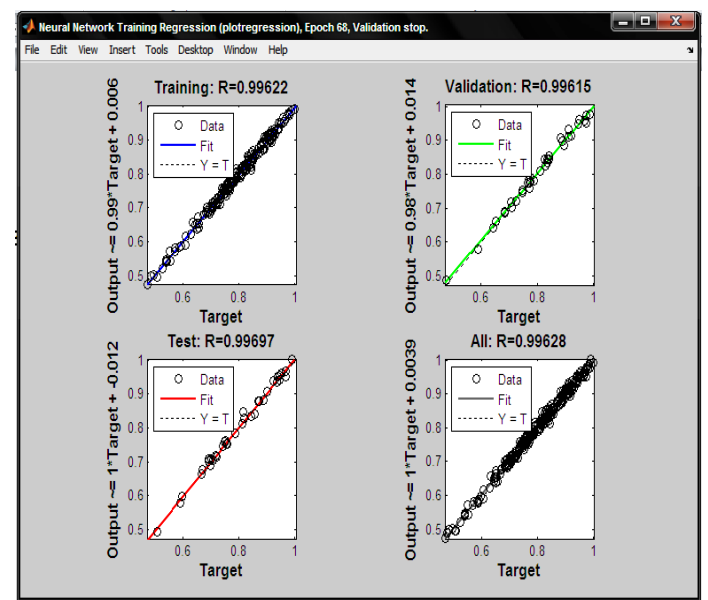

Fig. 9 ANC, Regression between enhanced and desired signal

$\mathrm{R}$ regression values illustrated in Fig. 9 are used to measure the correlation between the original and the enhanced signal. All curves have a high value greater than 0.99 . This means that the proposed algorithm has a good learning performance.

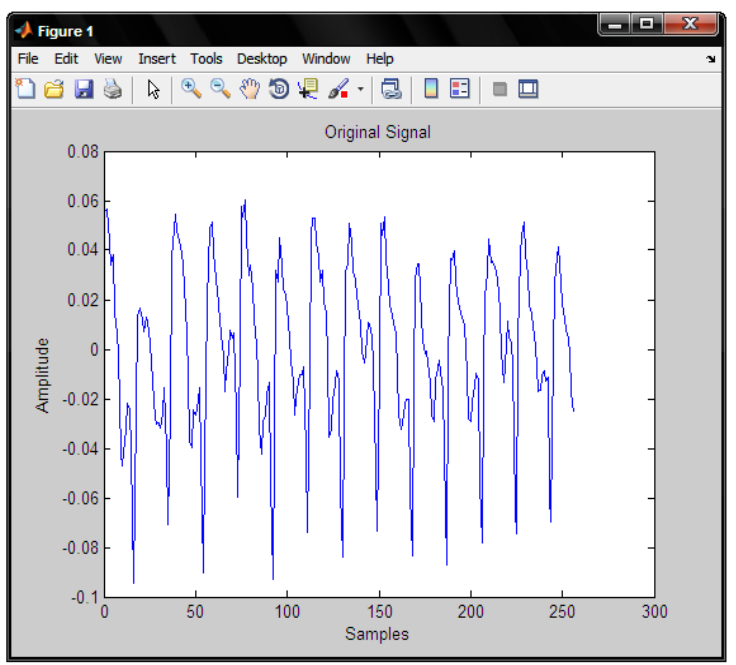

Fig. 10 Original signal used for neural ANC $(\mathrm{N}=256))$ 
Finally, the neural cancellation system's performance of the designed acoustic noise depends on the number of hidden layers and neurons in each hidden layer. Fig. 10 and 11 respectively represent the original signal and the noisy signal.

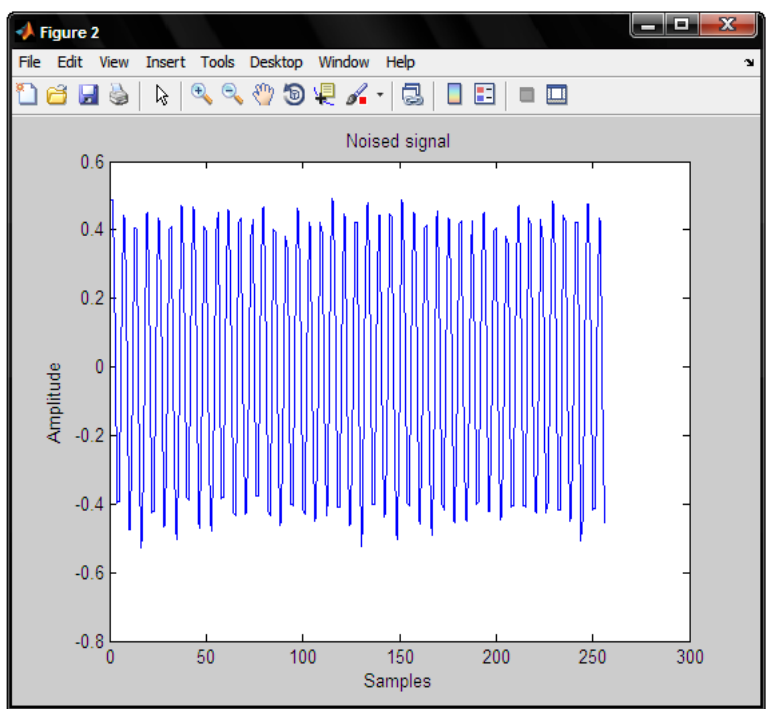

Fig. 11 Noised signal (neural Model), (Uniform noise + smooth signal, variance $\sigma=0.1, \mathrm{~N}=256$ )

While Fig. 12 represents the output of designed Embedded ANC system, also called the filtered or enhanced signal (signal without noise). During this work, to check the validity of the results obtained by the neural approach and allows us to compare the signal obtained by the network with the observed signal, we opted for the use of the signal to noise ratio (SNR) and the mean square error (MSE) [25].

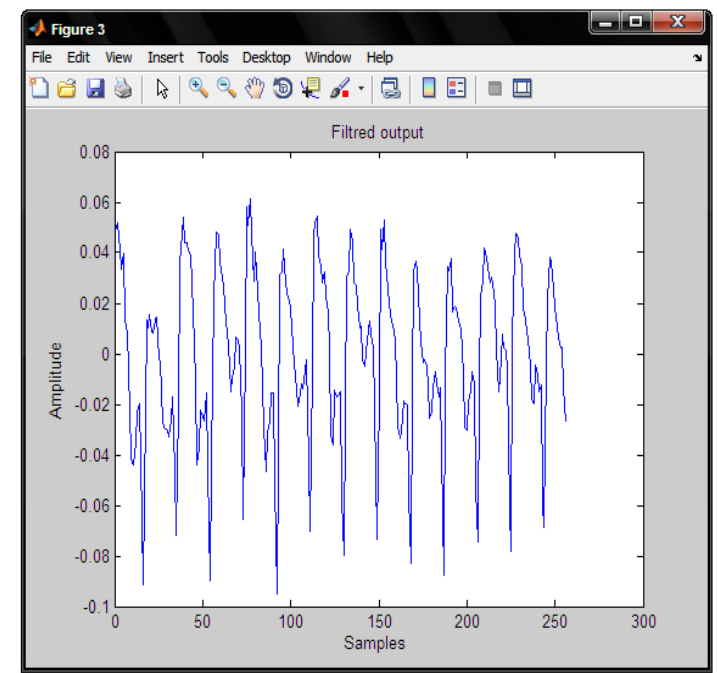

Fig. 12 Enhanced signal using neuronal ANC $(\mathrm{N}=256, \lambda=0.5$, neural architecture [10-5])

The following equations respectively give mathematical formulations:

$$
\begin{gathered}
S N R_{d B}=10 \log _{10}\left(\frac{\text { Power }(\text { originalSignal })}{\text { Power }(\text { Noise })}\right) \\
M S E=\frac{1}{2} \sum_{k}\left(d_{k}-y_{k}\right)^{2}
\end{gathered}
$$

Where, $\mathrm{d}_{\mathrm{k}}=$ noisy signal; $\mathrm{y}_{\mathrm{k}}=$ noise estimate; $\mathrm{k}=$ number of samples.

TABLE II

SNR AND MSE NUMBER OF ITERATION $(\mathrm{N}=256, \Sigma=1, \Lambda=0.5$, NEURAL ARCHITECTURE [10-5])

\begin{tabular}{ccccc}
\hline $\begin{array}{l}\text { Number of } \\
\text { iteration }\end{array}$ & $\begin{array}{c}\text { Train } \\
\text { time (s) }\end{array}$ & MSE & SNR & Gradient \\
\hline 14 & 01 & $9.40 \mathrm{E}-05$ & 29.5085 & $2.90 \mathrm{E}-05$ \\
22 & 01 & $7.35 \mathrm{E}-05$ & 29.4527 & $6.78 \mathrm{E}-05$ \\
24 & 01 & $8.44 \mathrm{E}-05$ & 29.4906 & $7.09 \mathrm{E}-05$ \\
28 & 01 & $9.35 \mathrm{E}-05$ & 29.4891 & $4.93 \mathrm{E}-05$ \\
30 & 03 & $9.55 \mathrm{E}-05$ & 29.6070 & 0.000176 \\
41 & 01 & $8.70 \mathrm{E}-05$ & 29.5415 & $1.07 \mathrm{E}-05$ \\
54 & 02 & 0.000103 & 29.5643 & 0.000243 \\
68 & 02 & $9.71 \mathrm{E}-05$ & 29.4880 & $1.11 \mathrm{E}-05$ \\
\hline
\end{tabular}

TABLE III

SNR AND MSE $(\mathrm{N}=256, \Sigma=1$, NEURAL ARCHITECTURE [10-5], $\Lambda=0.5)$

\begin{tabular}{cccll}
\hline $\begin{array}{l}\text { Adaptation } \\
\text { step } \lambda\end{array}$ & $\begin{array}{l}\text { Number of } \\
\text { iteration }\end{array}$ & $\begin{array}{l}\text { Train } \\
\text { time } \\
\text { (s) }\end{array}$ & SNR & MSE \\
\hline 0.1 & 30 & 01 & 29.6070 & $9.55 \mathrm{E}-05$ \\
0.2 & 15 & 01 & 29.6100 & 0.000121 \\
0.3 & 117 & 03 & 29.5312 & $8.22 \mathrm{E}-05$ \\
0.4 & 24 & 01 & 29.4906 & $8.44 \mathrm{E}-05$ \\
0.5 & 54 & 02 & 29.5643 & 0.000103 \\
0.01 & 41 & 01 & 29.5415 & $8.70 \mathrm{E}-05$ \\
0.02 & 48 & 01 & 29.5435 & 0.000119 \\
0.03 & 10 & $<01$ & 29.4302 & 0.000102 \\
0.04 & 12 & 01 & 29.4912 & $8.43 \mathrm{E}-05$ \\
0.05 & 68 & 02 & 29.4880 & $9.71 \mathrm{E}-05$ \\
0.001 & 10 & $<01$ & 29.6161 & 0.000112 \\
0.002 & 14 & 01 & 29.5085 & $9.40 \mathrm{E}-05$ \\
0.003 & 22 & 01 & 29.4527 & $7.35 \mathrm{E}-05$ \\
0.004 & 19 & 01 & 29.5719 & $9.80 \mathrm{E}-05$ \\
0.005 & 45 & 01 & 29.5152 & $6.69 \mathrm{E}-05$ \\
0.0005 & 15 & 01 & 29.5613 & 0.000101 \\
\hline
\end{tabular}

TABLE IV

SNR AND MSE NEURAL ARCHITECTURE (N=256, $\Lambda=0.5, \Sigma=1)$

\begin{tabular}{ccccc}
\hline $\begin{array}{c}\text { Neuronal } \\
\text { architecture }\end{array}$ & $\begin{array}{c}\text { Train } \\
\text { time (s) }\end{array}$ & $\begin{array}{c}\text { Number of } \\
\text { iterations }\end{array}$ & MSE & SNR \\
\hline$[10-2]$ & 01 & 16 & 0.00423 & 31.3898 \\
{$[10-3]$} & 01 & 21 & 0.000110 & 29.5902 \\
{$[10-4]$} & 01 & 28 & $7.49 \mathrm{E}-05$ & 29.4119 \\
{$[10-5]$} & 01 & 35 & $7.87 \mathrm{E}-05$ & 29.5171 \\
{$[10-6]$} & 01 & 45 & $9.39 \mathrm{E}-05$ & 29.4667 \\
\hline
\end{tabular}

\section{Discussion Results}

The tables below show the measurement results of MSE for evaluating performance concerning the artificial approach to improving the voice quality degraded by the acoustic noise. These values were obtained when the non-linear based on neural networks and filtering gave better results than when linear filtering based on adaptive approaches [26]-[28]. In this case, we worked on several neural structures with multiple learning functions. We realized that the architecture with two hidden layers $\{[10-5]\}$ is the most stable for all tests because it always converges to the optimal solution (global minimum). Finally, the ANN Embedded ANC System's performance depends on the number of hidden layers and the number of neurons in each hidden layer, learning but also used. 


\section{CONCLUSION}

In this work we proposed an artificial neural network Embedded Acoustic Noise Cancellation System for enhancing the quality of communication. The main advantage of the proposed system is its efficiency for non-linear phenomena such as acoustic noise. The proposed Embedded ANC's effectiveness is then evaluated by using SNR and MSE, neuronal architecture, and number of iteration criteria. However, the obtained result can show that the ANN ANC algorithm provides the fastest convergence rate, best values of SNR, and lowest MSE compared to adaptive algorithms. Further work could be done in implementing the embedded ANC for a real-time applications by using a Digital Signal Processor (DSP), Field Programmable Gate Array (FPGA) or Application-Specific Integrated Circuit (ASIC).

\section{REFERENCES}

[1] K. R. Borisagar et al., "Speech Enhancement Techniques for Digital Hearing Aids", Springer Nature Switzerland AG, 2019.

[2] Saeed V. Vaseghi, “Advanced Digital Signal Processing and Noise Reduction", John Wiley \& Sons, Inc. January 2006.

[3] Jingdong Chen et al., "Filtering Techniques for Noise Reduction and Speech Enhancement, Adaptive Signal Processing”, Springer, Berlin, Heidelberg, pp. 129-154, 2003.

[4] B. Moons et al., "Embedded Deep Learning", Springer Nature Switzerland AG, 2019

[5] M. Tanveer et al., "Machine Intelligence and Signal Analysis", Springer Verlag, Singapore, 1st ed, 2019

[6] J. Kapoor, G. R. Mishra, and M. Rai, "Adaptive Least Mean Square Noise Cancellation Model Using Various Fixed Coefficient Digital Filters .," Int. J. Adv. Sci. Technol., vol. 29, no. 10, pp. 8448-8455, 2020.

[7] T.J.Moir, "Adaptive crosstalk-resistant noise-cancellation using $\mathrm{H}$ infinity filters", IEEE International Conference on Signals and Systems (ICSigSys), pp. 123-456, 2019.

[8] M. T. Akhtar, "An adaptive algorithm, based on modified tanh nonlinearity and fractional processing, for impulsive active noise control systems," J. Low Freq. Noise, Vib. Act. Control, vol. 37, no. 3, pp. 495-508, Sep. 2018, doi: 10.1177/1461348417725952.

[9] Chang Liu et al., "Robust Adaptive Filter with Lncosh Cost", Signal Processing, volume 168, march, 107348, 2020.

[10] Rachana Nagal et al., "An Optimal Approach for Eeg/Erp Noise Cancellation Using Adaptive Filter with Oppositional Whale Optimization Algorithm", Biomedical Engineering: Applications, Basis and Communications, Vol. 31, No. 05, 1950035, 2019.

[11] D Niranjan et al., "Noise cancellation in musical signals using adaptive filtering algorithms", International Conference on Innovative Mechanisms for Industry Applications (ICIMIA), pp. 82- 86, 2017.

[12] Aniket Kumar et al., "Comparative research of various adaptive algorithms for noise cancellation in speech signals", International Conference on Control, Computing, Communication and Materials (ICCCCM) , pp. 1- 5, 2016.

[13] Azeddine Wahbi, Ahmed Roukhe, Laamari Hlou, "Modeling and Real-Time DSK C6713 Implementation of Normalized Least Mean
Square (NLMS) Adaptive Algorithm for Acoustic Noise Cancellation (ANC) In Voice Communications", (JATIT) Journal of Computer Technology \& Applications, Vol. 65, No.2, pp. 312-319, 2014.

[14] Azeddine Wahbi, Ahmed Roukhe, Laamari Hlou, "Conception and Real Time DSK C6713 of a Low Cost Adaptive Acoustic Noise Cancellation (ANC) Based Fast Fourier Transform (FFT) and Circular Convolution for Improving the Quality of Voice Communications", (IJCTA) International Journal of Computer Technology \& Applications, Vol 5, No.2, pp. 630-639, 2014.

[15] Azeddine Wahbi et al., "Modeling and Simulation of Recursive Least Square Adaptive (RLS) Algorithm for Noise Cancellation in Voice Communication", (JCC) Communication and Computer, Volume 10, issue 11. David Publishing Company, Vol. 11, pp. 1440-1444, 2013.

[16] Sheng Zhang, Jiashu Zhang, Hing Cheung So, "Mean square deviation analysis of LMS and NLMS algorithms with white reference inputs", Signal Processing ,131, pp. 20-26, 2017.

[17] Rodrigo M. S. Pimenta, Leonardo C. Resende, Newton N. Siqueira, Diego B. Haddad, Mariane R. Petraglia, "A New Proportionate Adaptive Filtering Algorithm with Coefficient Reuse and Robustness Against Impulsive Noise", 26th European Signal Processing Conference (EUSIPCO), pp. 470-474, 2018.

[18] Minajul Haque, Kaustubh Bhattacharyya, A study on different linear and non-linear filtering techniques of speech and speech recognition, ADBU Journal of Engineering Technology(AJET), Volume 8, Issue 1, June, 008010606 (6PP), 2019

[19] Maximilian Strake et al., "Fully Convolutional Recurrent Networks for Speech Enhancement, IEEE International Conference on Acoustics", Speech and Signal Processing (ICASSP), pp. 6674-6678, 2020.

[20] Han Zhao et al., "Convolutional-Recurrent Neural Networks for Speech Enhancement”, IEEE International Conference on Acoustics, Speech and Signal Processing (ICASSP), pp. 2401-2405, 2018.

[21] Andrew Maas et al., Recurrent Neural Networks for Noise Reduction in Robust ASR", 13th Annual Conference of the International Speech Communication Association, pp. 22-25, 2012.

[22] Ke Tan et al., "A Convolutional Recurrent Neural Network for RealTime Speech Enhancement, Interspeech, pp. 3229- 3233, 2018.

[23] Byoung-Tak Zhang, "An Incremental Learning Algorithm That Optimizes Network Size and Sample Size in One Trial”, IEEE International Conference on Neural Networks, Florida, pp. 215-220, 1994.

[24] D. E. Rumelhart, G. E. Hilton, and R. J. Williams, "Learning representations by back-propagation errors", Nature, 323, pp. 533-536, 1986.

[25] Noman Q. Al-Naggar and Mohammed H. Al-Udyni, "Performance of Adaptive Noise Cancellation with Normalized Last-Mean-Square Based on the Signal-to-Noise Ratio of Lung and Heart Sound Separation", Journal of Healthcare Engineering, Volume 2018.

[26] G.K Rajini et al., "A Research on Different Filtering Techniques and Neural Networks Methods for Denoising Speech Signals", International Journal of Innovative Technology and Exploring Engineering (IJTTEE) Volume-8, Issue-9S2, pp. 503- 513, 2019.

[27] Kuan-Chun Chen et al, "Active Noise Control In a Duct to Cancel Broadband Noise", 1st Nommensen International Conference on Technology and Engineering, IOP Conf. Series: Materials Science and Engineering, Volume 237, Issue 1, pp. 012015, 2017.

[28] R. Ram and M. N. Mohanty, "Fractional DCT ADALINE method for speech enhancement," 2017. 